\title{
microRNA-1271-5p/TIAM1 suppresses the progression of ovarian cancer through inactivating Notch signaling pathway
}

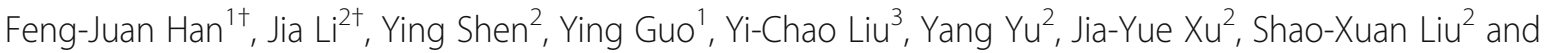
Yan-Hong Wang ${ }^{4^{*}}$ (D)

\begin{abstract}
Objective: Ovarian cancer (OC) has been regarded as the most malignant gynecological neoplasm and often confers grave outcomes owing to the frequent metastasis and high recurrence. A previous study has demonstrated that miR-1271-5p is implicated in OC progression, however, the possible mechanism of it remains unknown. The purpose of this investigation was to explore how miR-1271-5p regulates the progression of OC.

Methods: Gene Expression Omnibus (GEO) and The Cancer Genome Atlas (TCGA) databases were employed to analyze the differentially expressed miRNAs or genes as well as their corresponding prognostic values. miR-1271-5p expression in OC cells was examined by qRT-PCR. Cell counting kit 8 (CCK-8), colony formation, and transwell tests were conducted to evaluate the proliferation, migration and invasion potentials. Bioinformatics prediction and luciferase activity analysis were utilized to predict and verify the target gene of miR-1271-5p. Western blot assay was carried out to measure protein expression.

Results: miR-1271-5p was significantly decreased in $O C$ and its down-regulation was associated with the grave outcome of OC patients. Upregulation of miR-1271-5p inhibited cell viability, but miR-1271-5p knockdown promoted the proliferation of OC cells. TIAM1 was a direct target gene of miR-1271-5p and expressed in OC tissues at higher level. High expression of TIAM1 induced the poorer prognosis of patients with OC. Further functional analyses showed that the suppressive role of miR-1271-5p on OC cell malignant behaviors was overturned by the upregulation of TIAM1. The protein levels of Cyclin D1, HES1, NOTCH and NUMB were remarkably changed due to the abnormal expression of miR-1271-5p and TIAM1.

Conclusion: To sum up, miR-1271-5p inhibits proliferation, invasion and migration of OC cells by directly repressing TIAM1 to inactivate the Notch signaling pathway, which provides an alternative therapeutic candidate for the advancement of $O C$ treatment.
\end{abstract}

Keywords: miR-1271-5p, Ovarian cancer, Prognosis, Cell behavior, Notch pathway

\footnotetext{
*Correspondence: hljzyylj@163.com

${ }^{\dagger}$ Feng-Juan Han and Jia Li contributed equally to this work.

${ }^{4}$ Heilongjiang University of Traditional Chinese Medicine, College of

Pharmacy, No. 24, Heping Road, Xiangfang District, Harbin 150040,

Heilongjiang Province, China

Full list of author information is available at the end of the article
}

(C) The Author(s). 2020 Open Access This article is licensed under a Creative Commons Attribution 4.0 International License, which permits use, sharing, adaptation, distribution and reproduction in any medium or format, as long as you give appropriate credit to the original author(s) and the source, provide a link to the Creative Commons licence, and indicate if changes were made. The images or other third party material in this article are included in the article's Creative Commons licence, unless indicated otherwise in a credit line to the material. If material is not included in the article's Creative Commons licence and your intended use is not permitted by statutory regulation or exceeds the permitted use, you will need to obtain permission directly from the copyright holder. To view a copy of this licence, visit http://creativecommons.org/licenses/by/4.0/ The Creative Commons Public Domain Dedication waiver (http://creativecommons.org/publicdomain/zero/1.0/) applies to the data made available in this article, unless otherwise stated in a credit line to the data. 


\section{Background}

Ovarian cancer $(\mathrm{OC})$ is a principal reason of cancer death from gynecological malignancy, which accounts for $2.4-6.5 \%$ of tumors in females [1]. The status of OC in reproductive system cancers of women is second only to cervical cancer and endometrial cancer [2]. The surgery and radio-chemotherapy are the accustomed therapeutic strategies for OC [3]. Statistical data reveal that the five-year survival rate of all OC patients is only $47 \%$, even less than $20 \%$ in advanced stage patients [4]. Therefore, investigating the potential indicators for the early diagnosis and identifying efficacious targets are urgently required to be developed for $\mathrm{OC}$ patients.

miRNAs are endogenous short RNAs composed of 21-25 nucleotides involved in tumorigenesis $[5,6]$. Several studies suggest the crucial effect of miRNAs in OC development. miR-212-3p functions as a key target and suppresses the progression of high-grade serous $\mathrm{OC}$ via interacting with MAP 3 K3 [7]. The cyst formation and mode of OC spread are governed by miR-200 family [8]. Paliwal et al. illustrated that miR-22 and miR-21 are wonderful diagnostic indicators for OC early diagnosis [9]. miR-30b-3p as an anti-tumor gene in $\mathrm{OC}$ represses the migration and invasion of cells via down-regulating the CTHRC1 gene [10]. Among the massed miRNAs, miR-1271-5p is expressed at abnormal levels in many human cancers and participates in the tumorigenesis $[11,12]$. Of note, a ten-miRNA signature including miR-1271-5p has been identified from the genomewide miRNA expression profiling in OC [13]. This suggests that miR-1271-5p maybe a promising therapeutic molecule to treat OC. However, the potential effect of miR-1271-5p in human $\mathrm{OC}$ as well as its underlying molecular mechanism has been poorly understood.

TIAM1 (T cell lymphoma invasion and metastasis 1 ) is initially found in mice $\mathrm{T}$ lymphoma cells and considered as a metastasis-related gene [14]. Extensive evidences have illustrated that TIAM1, as a guanine nucleotide exchange factor, is involved in the invasion and metastasis of various human tumors, such as gastric cancer [15], prostate cancer [16] and breast cancer [17]. A previous report uncovered that TIAM1 expression is closely related with the grade and prognosis in OC and might be helpful for the management of clinic [18]. TIAM1 has also been indicated to facilitate the growth and invasion of OC cells through negatively being regulated by miR-22, miR-183 and miR-31 [19]. Despite that, the relevance between miR-1271-5p and TIAM1 in OC still remain elusive.

Recently, Notch signaling pathway has aroused more attention for carcinogenesis, which acts as an evolutionarily conserved pathway involved in the regulation of multiple biological processes, including proliferation, apoptosis, invasion and differentiation $[20,21]$. The Notch family is composed of four receptors and five ligands [22]. Notably, Notch pathway has been reported to affect the progression of different kinds of cancers [23]. TIAM1 was positively related with the Notch signaling pathway. Furthermore, an investigation indicated that a crosstalk exists between miRNAs and Notch pathway in multiple malignancies [24].

Herein, this work aimed to evaluate the biological role of miR-1271-5p in OC, so as to decipher the prospective mechanism and its prognostic value. All results showed that miR-1271-5p might exert a suppressive role on the progression of $\mathrm{OC}$, and we also discovered that miR1271-5p/TIAM1 axis regulates the development of OC through mediating the Notch signaling pathway. These findings can advance our understanding of OC progression and potential treatment options for OC.

\section{Methods}

Tissue samples

Gene Expression Omnibus (GEO; https://www.ncbi.nlm. nih.gov/geo/) is an open public genomics repository and used to help researchers inquire and download gene expression profiles. The data set GSE119055 derived from GEO was prepared to show the difference of miR-1271$5 \mathrm{p}$ expression level in normal controls $(n=3)$ and OC tissue samples $(n=6)$. Expression of TIAM1 in OC and its prognostic value were analyzed using TCGA-OC cohort (https://cancergenome.nih.gov/) that included 378 OC tissue samples. The normal specimens $(n=88)$ were provided by Genotype-Tissue Expression (GTEx) project (http://commonfund.nih.gov/GTEx/).

\section{Cell culture and transfection}

Human normal control cell line IOSE80 (SXBIO Co., Ltd., Shanghai, China) and human OC cell lines (SK-OV-3, Cell Biology of the Chinese Academy of Sciences, Shanghai, China; OVCAR-3, American Type Culture Collection, Manassas, VA, USA) were incubated in Dulbecco's Modified Eagle's Medium (DMEM, Gibco, Grand Island, NY, USA) supplemented with $10 \%$ fetal bovine serum (FBS, Gibco) and specific antibiotics $(100 \mathrm{U} / \mathrm{mL}$ penicillin and $0.1 \mathrm{mg} / \mathrm{mL}$ streptomycin) at $37{ }^{\circ} \mathrm{C}$ with $5 \% \mathrm{CO}_{2}$. miR-1271-5p mimic (5' -CUU GGC ACC UAG CAA GC A CUC A-3'), inhibitor (5'-UGA GUG CUU GCU AGG U GC CAA G-3') and scrambled miR-negative control (NC; 5'-UCA CAA CCU CCU AGA AAG AGU AGA-3'); small inference RNAs against TIAM1 (si-TIAM1\#1 and siTIAM1\#2) and corresponding NC (si-con) were synthesized by Genepharma Co., Ltd. (Shanghai, China). Full length TIAM1 was cloned into the pcDNA3.1 vector and the resultant was named as pcDNA3.1-TIAM1 (TIAM1). Its negative control was named as pcDNA3.1-empty vector (vector). Cells were transfected with above agents using Lipofectamine2000 (Life Technologies, USA). Twenty-four hours later, the transfection efficiency was measured with qRT-PCR test. 


\section{RNA isolation and qRT-PCR}

qRT-PCR was performed to measure the expression of miR-1271-5p and TIAM1 in OC cells. Firstly, TRIZOL solution was applied to isolate the total RNA of transfected OVCAR-3 and SK-OV-3 cells which was then reverse transcribed into cDNA by the PrimeScript RT reagent Kit (Takara, Dalian, China). SYBR Premix Ex Taq II was applied for the performance of qRT-PCR on ABI 7500 PCR system (Foster, CA, USA). miR-1271-5p and TIAM1 expression was normalized to U6 or GAPD $\mathrm{H}$, respectively. Quantification of miR-1271-5p and TIAM1 expression was determined by $2^{-\Delta \mathrm{Ct}}$ method. Primers of this present study are revealed in Table 1.

\section{Protein extraction and western blot}

Western blotting was used to detect the expression levels of indicated proteins. After $24 \mathrm{~h}$ transfection, RIPA buffer supplemented with protease inhibitor PMSF was utilized to isolate proteins from OVCAR-3 and SK-OV-3 cells. The bicinchoninic acid (BCA) quantification kit (MultiSciences Biotech Co., Ltd., Shanghai, China) was applied to quantify the concentration of proteins. Separated proteins were firstly denatured at $95^{\circ} \mathrm{C}$ for $5 \mathrm{~min}$. Equal quantities of denatured protein $(20 \mu \mathrm{g})$ were loaded in $12 \%$ SDS-PAGE and electrophoretically transferred onto the PVDF membranes. PVDF membranes were sealed with $5 \%$ skimmed milk for $1 \mathrm{~h}$ at $37^{\circ} \mathrm{C}$, and subsequently incubated with specific primary antibodies at $4{ }^{\circ} \mathrm{C}$ overnight and HRP-tagged secondary antibody at $37^{\circ} \mathrm{C}$ for $1 \mathrm{~h}$. Following washing membranes with PBST, the protein signals were probed wit the ECL reagent. The protein signal was analyzed by ImageJ software.

\section{Cell viability analysis}

The proliferation capacity of OVCAR-3 and SK-OV-3 cells was elevated using CCK- 8 assay. Briefly, transfected cells (1000 cells/well) were seeded into a 96-well plate and cultured at $37^{\circ} \mathrm{C}$ with $5 \% \mathrm{CO}_{2}$ for $0,24,48$ and 72 h. Then, $10 \mu \mathrm{L}$ of CCK-8 solution was put into every well for additional $1.5 \mathrm{~h}$ incubation. Afterwards, a microplate reader was utilized to detect the OD values under

Table 1 Sequences of primers used in this study

\begin{tabular}{ll}
\hline Primer name & Sequence \\
\hline miR-1271-5p & F: 5'- TTGGCACCTAGCAAGCA -3' \\
& R: 5'-GAACATGTCTGCGTATCTC-3' \\
U6 & F: 5'-CGCAAATTCGTGAAGCGTTC-3' \\
& R: 5'-TTGCGTGTCATCCTTGCG -3' \\
TIAM1 & F: 5'- AAATCACACGGCGACCTGTCGT -3' \\
& R: 5'- ATGGCATCCTGAAGCCTCATCC - 3' \\
GAPDH & F: 5'- GTCAAGGCTGAGAACGGGAA - 3' \\
& R: 5'- AGTGATGGCATGGACTGTGG - 3' \\
\hline
\end{tabular}

$450 \mathrm{~nm}$ to assess the cell viability at predefined time points.

\section{Colony formation}

The clonogenicity of $\mathrm{OC}$ cells was measured by colony formation experiments. After specific transfection, 1000 cells per well were inoculated into the six-well plates and cultured in DMEM medium with $10 \%$ FBS at $37^{\circ} \mathrm{C}$ with $5 \% \mathrm{CO}_{2}$ for two weeks. Next, colonies observed by naked eyes were fixed in $4 \%$ paraformaldehyde and dyed in $0.1 \%$ crystal violet for $30 \mathrm{~min}$, respectively. Washed the staining reagent gently using running water and artificially counted the number of colonies with more than 50 cells under a microscope. Three plates were seeded for each cell line and this experiment was repeated on three different days.

\section{Transwell test}

The migration and invasion capabilities of OVCAR-3 and SK-OV-3 cells were examined using a transwell test. The upper surface of transwell chambers pre-coated with Matrigel was employed to detect invasion ability, and the migration capacity detection required no Matrigel. After $24 \mathrm{~h}$ transfection, OC cells $\left(1 \times 10^{5}\right)$ were placed in the top chamber with $100 \mu \mathrm{L}$ serum-free medium; simultaneously, culture medium $(500 \mu \mathrm{L})$ was added into the bottom chamber. Followed by overnight incubation, cells remain on the upper surface were removed using the cotton swabs and the migrated or invaded cells in the lower transwell chamber were fixed in $4 \%$ paraformaldehyde and dyed with $0.1 \%$ crystal violet for $30 \mathrm{~min}$, respectively. Cells were washed using PBS for three times in triplicates. Five random fields were picked and the numbers of migrated or invaded cells were counted under a microscope.

\section{Dual-luciferase activity assay}

In order to verify the candidate targets, luciferase activity assay was implemented. The specific dual-luciferase reporter vectors (wild type-TIAM1 and mutant-TIAM1) were synthesized and transfected into OC cells by using Lipofectamine2000 according to the guidelines of manufacturer. Forty-eight hours later, transfected cells were collected for the measurement of the luciferase activity by a dual-luciferase reporter assay kit (Promega, Madison, WI, USA).

\section{Statistical analysis}

All experiments were done in triplicates, and each test was repeated three times. Results were statistically analyzed using SPSS22.0 software and GraphPad Prime 8.0, and exhibited as mean \pm SD. Student's t-test and ANOVA was performed to compare the differences in two or more groups. Kaplan-Meier method was utilized 
to plot the overall survival curve. The relationship between miR-1271-5p and TIAM1 in OC was identified using Pearson's correlation analysis. Gene set enrichment analysis (GSEA) enrichment was performed with GSEA (version2.2.2, http://www.broadinstitute.org/gsea). $P<0.05$ was regarded as significance.

\section{Results}

Downregulation of miR-1271-5p in OC is related with unfavorable prognosis and elevates cell viability

In order to explore the possible therapeutic targets that exert crucial effects in OC development, we firstly screened the differential expression miRNAs in GSE119055 by using R package "limma" with FDR $<0.05$ and $\mid \log$ (FC) $\mid \geq 2$. Analysis showed that a total of 25 miRNAs were expressed with lower levels in OC tissue samples compared with normal controls. Subsequently, the prognostic power of these 25 down-regulated miRNAs was analyzed with the threshold of $P<0.05$ and FDR $<0.05$ based on TCGA-OC cohort. Finally, three miRNAs including miR-1271-5p, miR-501-3p and miR-500a-3p were identified differential expression miRNAs with potential prognostic value in OC. Notably, miR-1271-5p ranked the second lowest based on the fold change. Thus, based on the comprehensive literature analysis, miR-1271-5p was picked for future experimental analysis. Figure 1a and b showed that miR-1271-5p was declined in OC tissues $(P=0.0001)$ and cell lines $\left({ }^{* * *} P<\right.$ 0.01). On the basis of the TCGA-OC cohort, OC clinical samples were assigned into high-miR-1271-5p group and low-miR-1271-5p group owing to the median value of miR1271-5p expression. OC patients with a lower level of miR1271-5p had poorer outcomes compared to that with high miR-1271-5p level (Fig. 1c, $P=0.00555$ ). CCK-8 assay was carried out to measure the proliferation potential of $\mathrm{OC}$ cells (Fig. $1 \mathrm{~d}$ and e, ${ }^{* * *} P<0.01$ ). Results disclosed that the inhibition of miR-1271-5p expression promoted the proliferation, whereas overexpression of miR1271-5p repressed cell viability, in both OVCAR-3 and SK-OV-3 cells. These data indicated that miR-1271-5p might act as a suppressive factor in OC development.

\section{TIAM1 is a direct target of miR-1271-5p}

To investigate the target gene of miR-1271-5p in OC, we firstly clustered the TCGA-OC cohort and normal specimens from $G_{T E}$ database to screen the differentially expression genes in $\mathrm{OC}$, with $\mathrm{FDR}<0.05$ and $|\log \mathrm{FC}|>$ 2. A total of 2254 differentially expression genes were obtained, including 1018 upregulated genes and 1236 down-regulated genes. Target prediction tool suggested that there were 56 putative targets of miR-1271-5p in OC. A common gene TIAM1 was ultimately obtained after intersecting upregulated genes and candidate target genes (Fig. 2a). Compared with normal samples, TIAM1 was expressed at higher levels in OC tissues (Fig. $2 \mathrm{~b}, P<$
0.001). The survival curve showed that high expression of TIAM1 was related with unfavorable prognosis compared with OC patients carrying low level of TIAM1 (Fig. 2c, $P=0.02736$ ). Figure $2 d$ manifested the binding site between miR-1271-5p and TIAM1. Results of luciferase reporter gene analysis demonstrated that the transfection of miR-1271-5p mimic attenuated the luciferase activity of WT TIAM1 group, whilst miR-1271-5p inhibitor markedly increased the luciferase activity, in both OVCAR-3 and SK-OV-3 cells. Simultaneously, in the OVCAR-3 and SK-OV-3 cells, the transfection of miR1271-5p mimic or inhibitor exerted no impacts on the luciferase activity of MUT TIAM1 group (Fig. $2 \mathrm{~d},{ }^{* * *} P<$ 0.01). In addition, Pearson's correlation analysis confirmed that there was a negative relationship between miR-1271-5p and TIAM1 expression in OC (Fig. 2e, $P<$ 0.0001, $r=-0.4066)$. qRT-PCR and western blot experiments verified the correlation between miR-1271-5p and TIAM1 expression in OC cells (Fig. $3,{ }^{* *} P<0.01,{ }^{\# \#} P<$ $\left.0.01,{ }^{\& \&} P<0.01\right)$. In OVCAR-3 cells, miR-1271-5p inhibitor transfection increased the expression of TIAM1, whilst the addition of si-TIAM1 reversed the effect of miR-1271-5p inhibitor on TIAM1 expression (Fig. 3a and c, $\left.{ }^{* * *} P<0.01,{ }^{\# \#} P<0.01,{ }^{\& \&} P<0.01\right)$. By contrast, miR-1271-5p enhancement inhibited TIAM1 expression, which was rescued by the transfection of pcDNA3.1TIAM1 in SK-OV-3 cells (Fig. 3 b and d, $P<0.01,{ }^{\# \#} P<$ $\left.0.01,{ }^{\& \&} P<0.01\right)$. All observations uncovered that miR1271-5p can directly target TIAM1 and negatively regulated the expression of TIAM1 in OC.

miR-1271-5p suppresses the proliferation, migration and invasion capacities of OC cells via targeting TIAM1

To in-depth assess the biological role of miR-1271-5p/ TIAM1 in OC cellular malignant behaviors, OVCAR-3 cells were transfected with miR-1271-5p inhibitor, siTIAM1 and miR-1271-5p inhibitor + si-TIAM1; SKOV-3 cells were transfected with miR-1271-5p mimic, TIAM1 and miR-1271-5p mimic + TIAM1. CCK-8 results revealed that silencing TIAM1 suppressed the OVCAR-3 cell proliferation, and TIAM1 enhancement promoted cell viability of SK-OV-3 cells. Co-transfection of inhibitor + si-TIAM1 or mimic + TIAM1 was enable to overturn the individual effect induced by inhibitor, siTIAM1 or mimic, TIAM1 on cell proliferation (Fig. 4a and $\left.\mathrm{b},{ }^{* * *} P<0.01,{ }^{\# \#} P<0.01,{ }^{\& \&} P<0.01\right)$. Moreover, the colony formation test determined that the TIAM1 knockdown could abolish the elevating effect of miR1271-5pinhibitor on clonogenic ability in OVCAR-3 cells, and upregulation of TIAM1 could recover the miR-1271-5p-induced suppressive role on colony formation of SK-OV-3 cells (Fig. $4 \mathrm{c}$ and $\mathrm{d},{ }^{* *} P<0.01$, ${ }^{\# \#} P<$ $\left.0.01,{ }^{\& \&} P<0.01\right)$. The consistent results were presented in transwell migration and invasion analyses (Fig. 4e and 


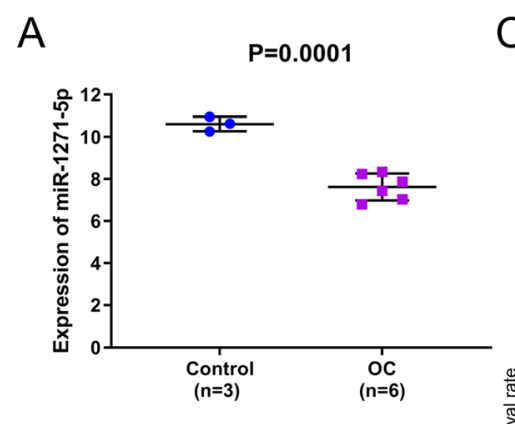

C

\section{B}
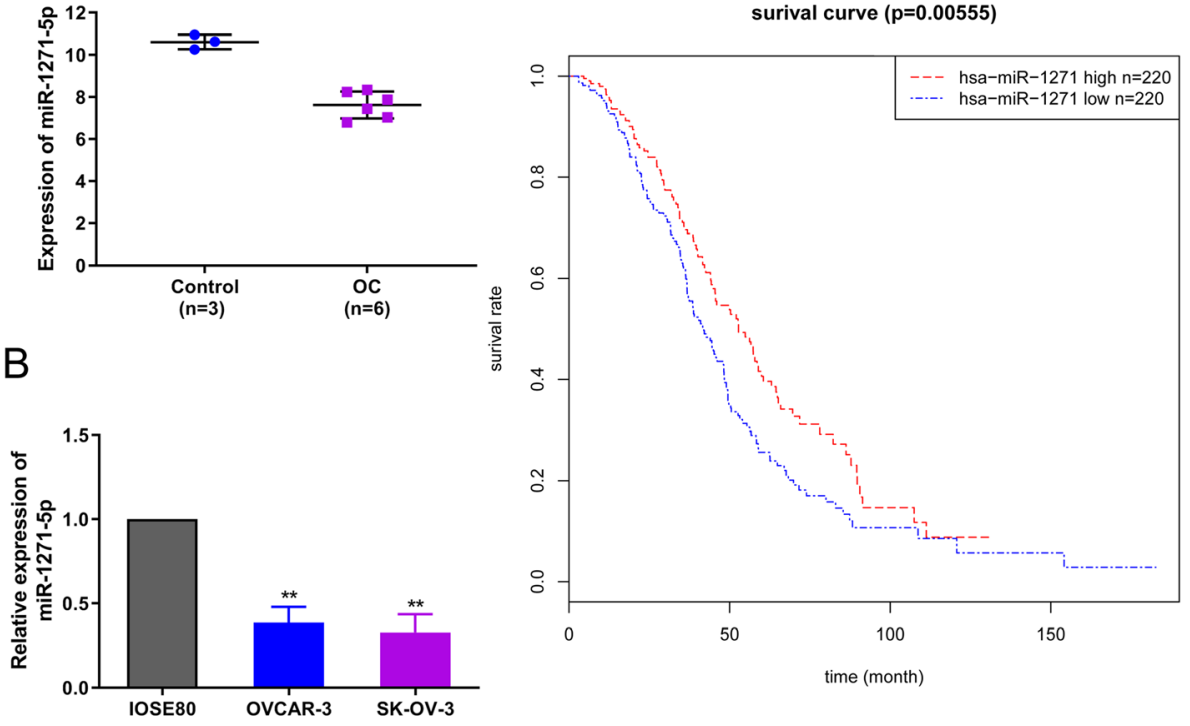

D
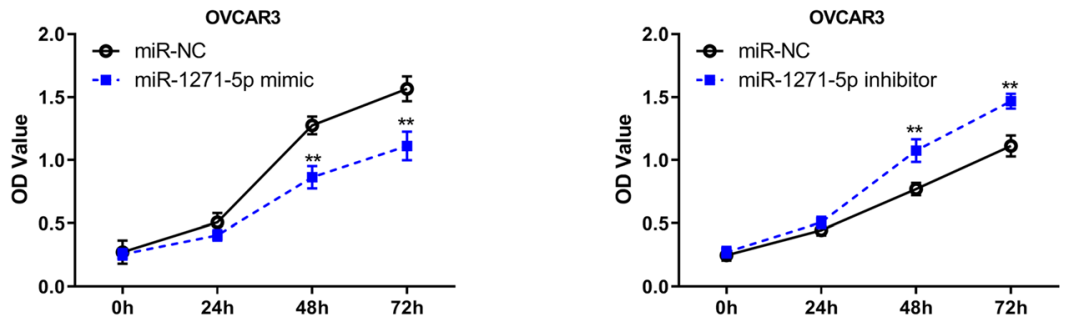

$E$
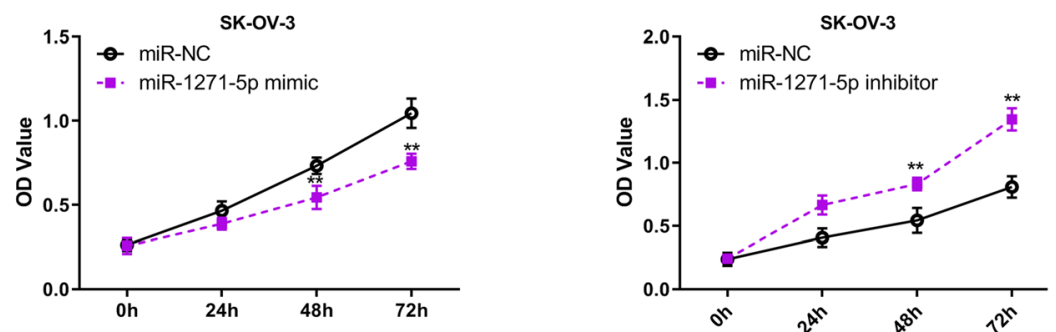

Fig. 1 miR-1271-5p was decreased in OC and affected the prognosis of patients with OC. a The expression level of miR-1271-5p in normal control $(n=3)$ and OC tissue samples $(n=6)$ based on the GSE1 19055 array, $P=0.0001$. b RT-PCR assay was implemented to detect the expression level of miR-1271-5p in different cell lines, ${ }^{* *} P<0.01$ versus IOSE80 cell line. $\mathbf{c}$ Kaplan-Meier curve of miR-1271-5p expression in OC of TCGA-OC cohort, $P=0.00555$. d-e The proliferation capacity of OVCAR-3 and SK-OV-3 cells were measured using CCK- 8 assay, ${ }^{* *} P<0.01$ versus miR-NC group

f, $\left.{ }^{* *} P<0.01,{ }^{\# \#} P<0.01,{ }^{\& \&} P<0.01\right)$. To summarize, all data illustrated that miR-1271-5p might function as a tumor-suppressive factor in OC through targeting TIAM1.

miR-1271-5p/TIAM1 axis regulates the activity of notch signaling pathway in $O C$

To explore how miR-1271-5p/TIAM1 axis regulates the progression of OC, GSEA enrichment analysis was performed and disclosed that TIAM1 was enriched in Notch signaling pathway. Next, we conducted western blot experiment to examine the effect of miR-1271-5p/ TIAM1 axis on the Notch pathway. Results of western blot were shown in Fig. 5. As exhibited in Fig. 5a, miR1271-5p inhibitor induced a increase level of Cyclin D1, HES1, and NOTCH, while declined the protein level of NUMB in OCVAR-3 cells. On the contrary, silencing TIAM1 attenuated the proteins levels of Cyclin D1, 


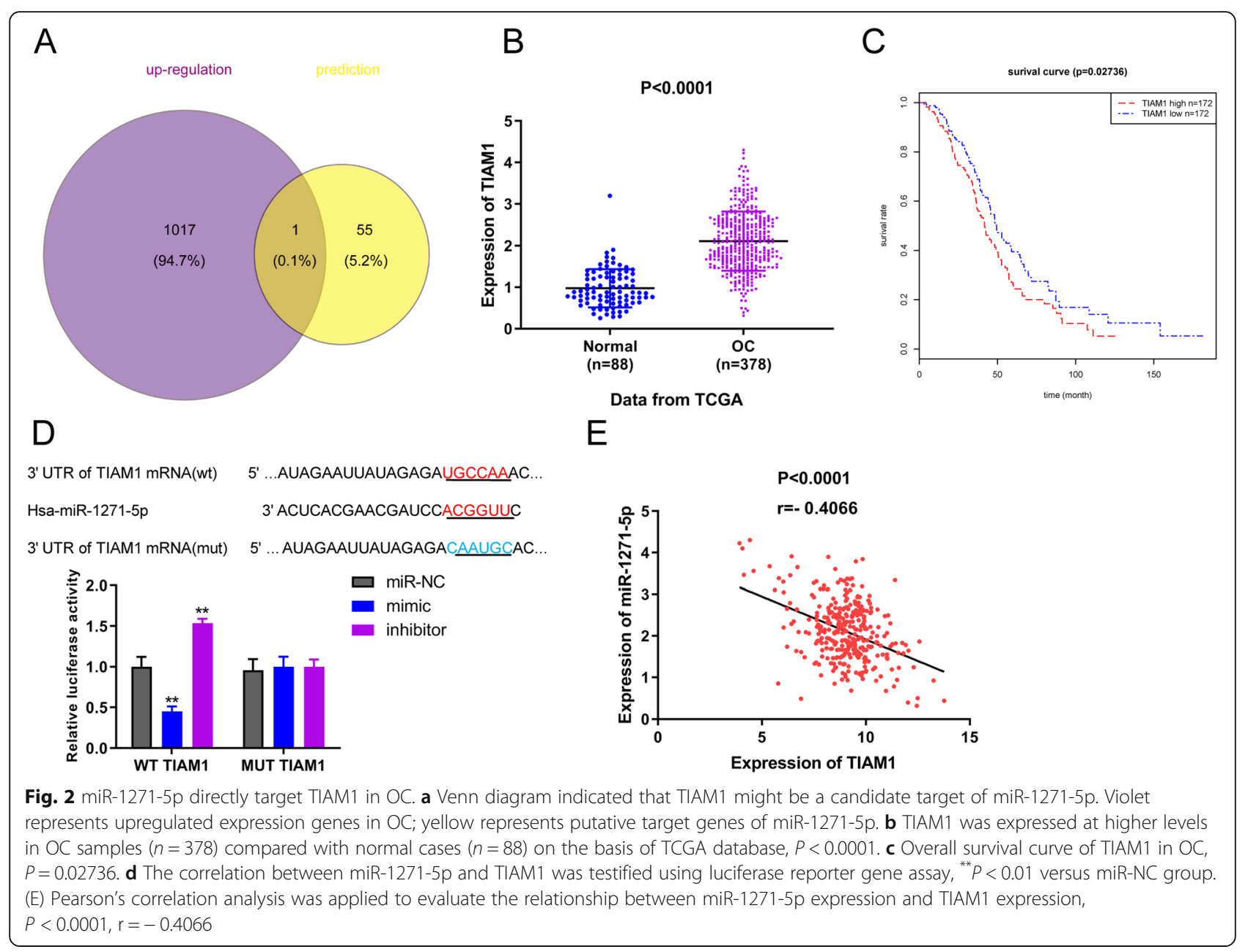

HES1, and NOTCH, elevated NUMB level. Interestingly, co-transfection miR-1271-5p inhibitor and siTIAM1 recovered these proteins levels that significantly changed by miR-1271-5p inhibitor or si-TIAM1 (Fig. 5a, $\quad P<0.01,{ }^{\# \#} P<0.01, \quad \& \& P<0.01$ ). On the other hand, the results in SK-OV-3 cells revealed that overexpression of miR-1271-5p led to a lower level of Cyclin D1, HES1, and NOTCH, whereas NUMB was markedly increased. The opposite tendency of these proteins was caused by TIAM1 enhancement. Cotransfection of miR-1271-5p mimic+TIAM1 remarkably restored the expression levels of Cyclin D1, HES1, NOTCH, and NUMB to normal levels (Fig. 5b, $\left.{ }^{* * *} P<0.01,{ }^{\# \#} P<0.01,{ }^{\& \&} P<0.01\right)$. Taken together, miR1271-5p/TIAM1 axis regulates the progression of OC via mediating the activity of Notch signaling pathway.

\section{Discussion}

Former studies have demonstrated the important regulatory role of miR-1271-5p in numerous cancers. For instance, miR-1271-5p can repress cell viability and promote radiosensitivity through targeting CDK1 in hepatocellular carcinoma [25]. miR-1271-5p, sponged by lncRNA UCA1, contributes to apoptosis and inhibits the proliferation capacity in multiple myeloma [26]. Chen et al. indicated that miR-1271 blocks the development of papillary thyroid carcinoma via inhibiting IRS1 expression and inactivating the AKT signaling pathway [27]. Importantly, miR-1271 has been reported as a suppressive factor in OC cells, which suppresses growth and invasion through mediating epithelialmesenchymal transition [28]. However, the specific molecular mechanism of miR-1271-5p in OC still needed to be detected. In our present study, aberrant miR1271-5p expression affected the proliferative ability of OC cells. All results identified the inhibitory role of miR-1271-5p in OC cellular behaviors. Downregulation of miR-1271-5p was associated with unfavorable prognosis of OC patients.

miRNAs are enable to regulate physiological and pathological processes through repressing or promoting target mRNAs translation. To further explore the 

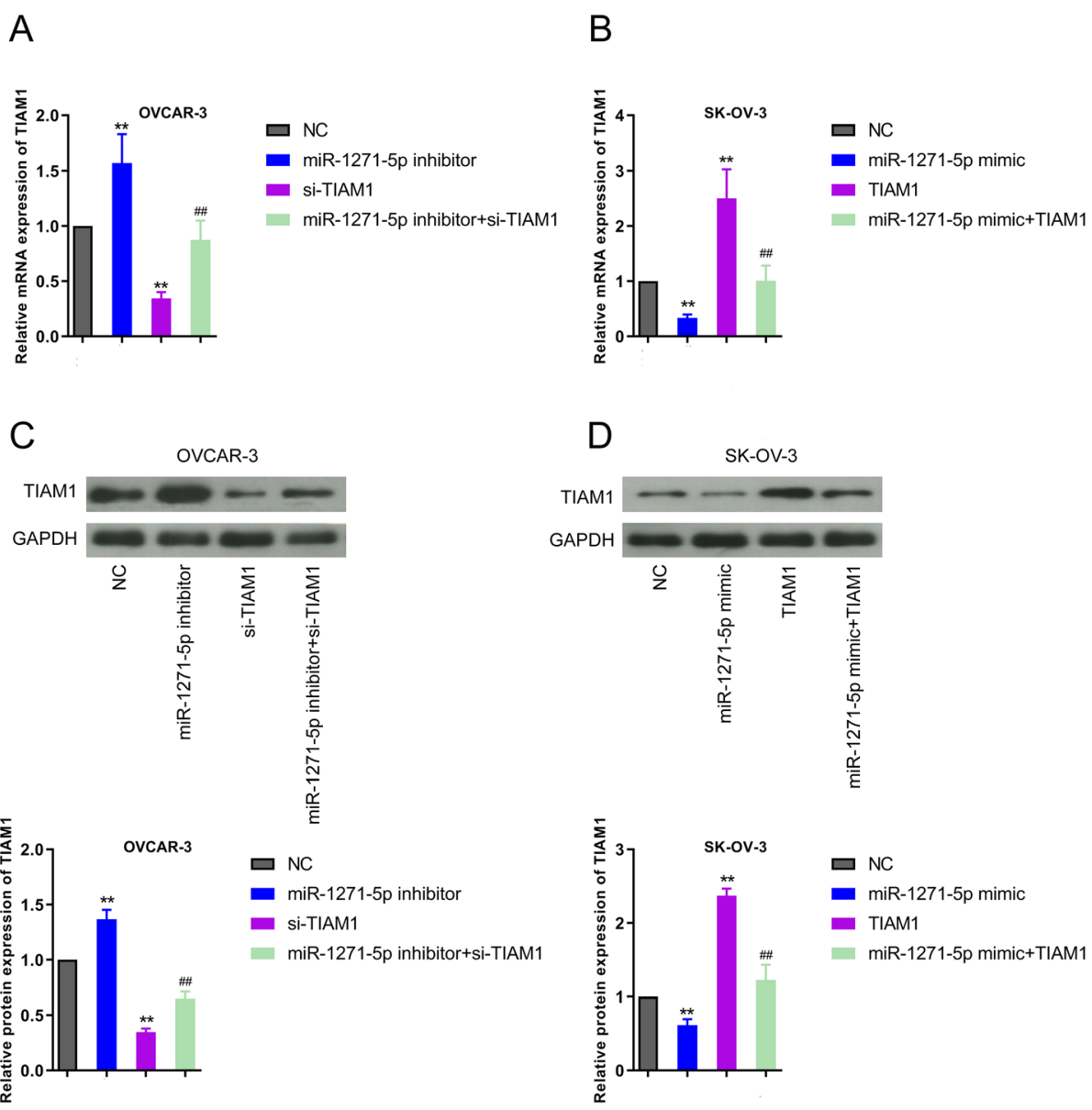

Fig. 3 TIAM1 expression was negatively regulated by miR-1271-5p. a-b Relative mRNA expression of TIAM1 was assessed by qRT-PCR in OVCAR-3 and SK-OV-3 cells after different transfections, including inhibitor/mimic, si-TIAM1/TIAM1, inhibitor+Si-TIAM1 or mimic+TIAM1, ${ }^{* *} P<0.01$ versus NC group, ${ }^{\# \#} P<0.01$ versus inhibitor or mimic group, ${ }^{\&}{ }^{\&} P<0.01$ versus si-TIAM1 or TIAM1 group. $\mathbf{c}-\mathbf{d}$ After above transfections, western blot experiment was performed to measure the protein expression level of TIAM1 in OVCAR-3 and SK-OV-3 cells. The gray values of protein bands were quantified. ${ }^{* *} P<0.01$ versus NC group, ${ }^{\# \#} P<0.01$ versus inhibitor or mimic group, ${ }^{\&} \&<0.01$ versus si-TIAM1 or TIAM1 group

underlying molecular mechanism of miR-1271-5p in $\mathrm{OC}$, we screened the differentially expressed genes according to the TCGA-OC cohort and $\mathrm{GTE}_{\mathrm{X}}$ database and intersected these upregulated genes with putative target genes of miR-1271-5p. Finally, a common gene TIAM1 was achieved. Here, in this investigation, TIAM1 was identified as a direct target gene of miR1271-5p in OC for the first time. Consistent with the previous publication, TIAM1 expression was dramatically increased in $\mathrm{OC}$ tissues and correlated with outcomes of OC patients. Its expression was negatively regulated by the expression of miR-1271-5p. Furthermore, we also found that TIAM1 played an opposite role with miR-1271-5p in the malignant behaviors of OC cells; TIAM1 overexpression rescued the cell viability, invasion and migration that abolished by miR-1271-5p mimic. In summary, these results depicted that miR-1271-5p/TIAM1 axis may exert an important role in the progression of OC.

Notch signaling pathway plays essential roles in the progression of OC and is active in human OC [29]. Besides, TIAM1 expression positively correlated with the activity of Notch signaling pathway in OC. Additionally, several reports indicate the highly relationship between miRNAs and Notch pathway. For example, the regulatory action of miR-27a, miR-139-5p and miR-433 in different tumors is related with the Notch pathway [3032]. The epigenetic silencing of miR-199b-5p has also been proven to be linked with the activation of Notch signaling in OC [33]. Thus, to verify whether the biological effect of miR-1271-5p/TIAM1 axis in OC cells is associated with the activity of Notch signaling pathway, we measured the levels of related proteins including Cyclin D1, HES1, Notch and NUMB by western blot. 


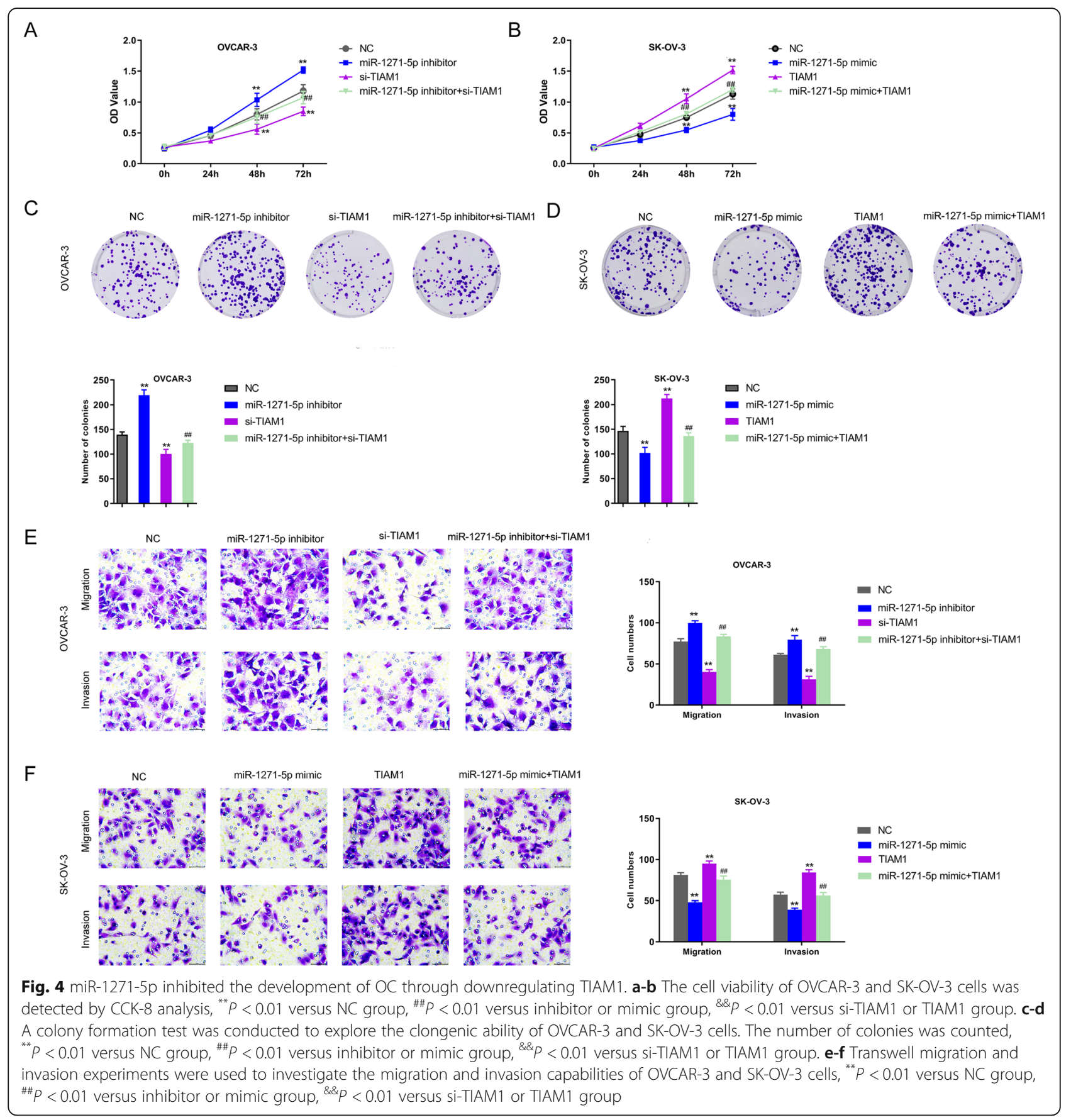

Notch1 is a highly conserved member of Notch family and exerts a vital role in various cancers [34]. Increased Notch1 expression is correlated with unfavorable prognosis of OC patients [35]. HES1 and Cyclin D1 are the downstream genes of Notch1 [36]. NUMB, as a Notch signaling pathway inhibitor, can suppress the activity of Notch signaling via directly binding to the domain of NICD [37]. According to the western blot results, the increased activity of Notch pathway was found in OVCAR-3 cells after miR-1271-5p inhibitor treatment; whereas TIAM1 knockdown inactivated the Notch pathway; co-transfection of miR1271-5p inhibitor and si-TIAM1 reversed the status of Notch pathway changed by miR-1271-5p inhibitor or si-TIAM1. Similarly, in SK-OV-3 cells, the combination of miR-1271-5p mimic and TIAM1 recovered the activity of Notch pathway to normal status. Collectively, these findings elucidated that miR-1271-5p/ 


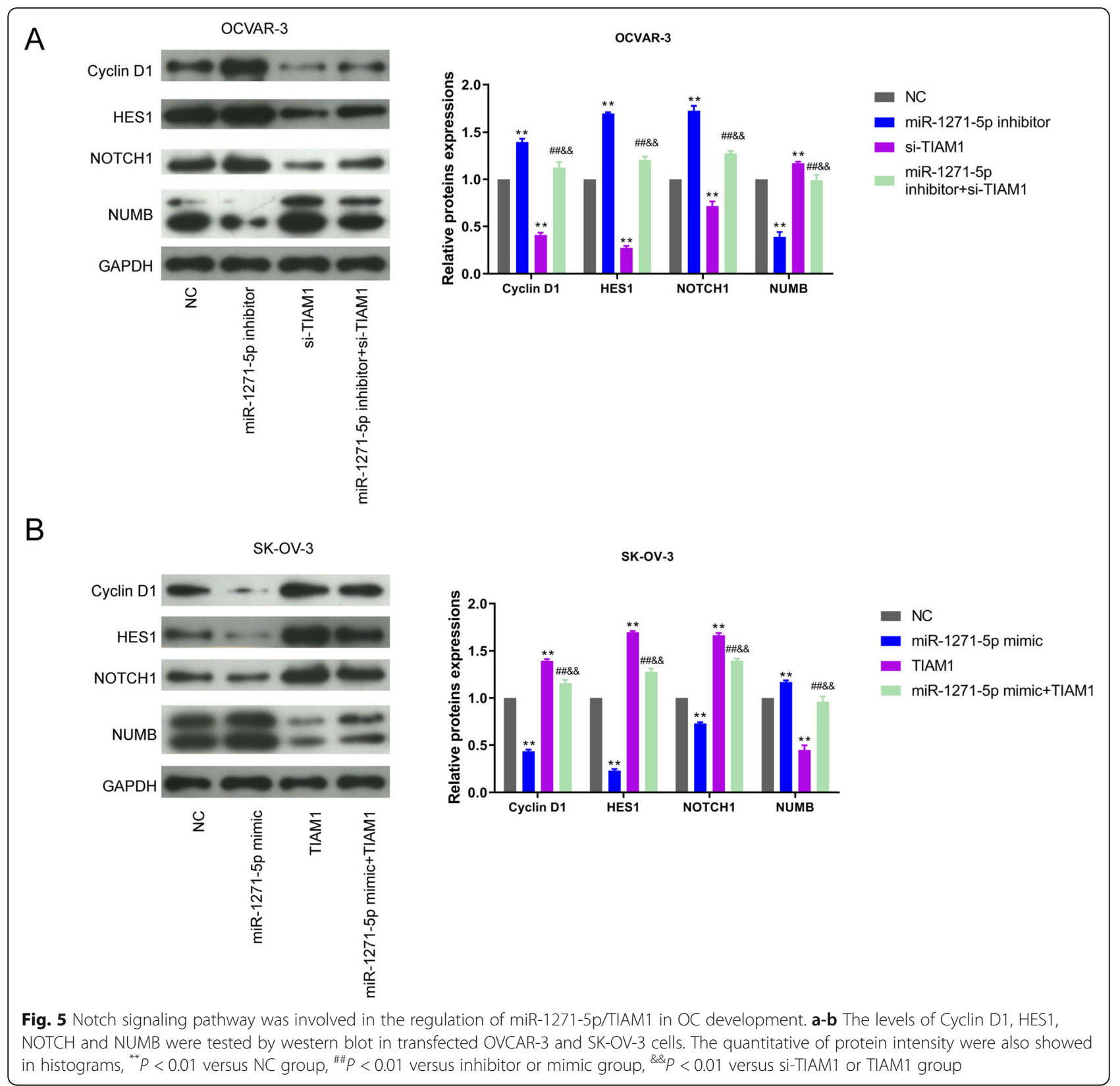

TIAM1 axis might regulate the progression of OC through mediating Notch signaling.

However, there are some limitations: due to the shortage of clinical samples downloaded from GEO datasets, we might have magnified the difference of miR-1271-5p expression and its biological function in OC. Therefore, it is necessary to collect the sufficient clinical specimens to validate the suppressive role of miR-1271-5p in the development of OC. In addition, in vivo experiments also required for in-depth validation. Whether Notch signaling pathway is directly or indirectly implicated in the regulatory signaling axis
miR-1271-5p/TIAM1 in OC still needs to be evaluated.

In conclusion, the expression of miR-1271-5p is significantly decreased in human $\mathrm{OC}$ and down-regulation of miR-1271-5p is associated with poorer outcomes of OC patients. Mechanically, miR-1271-5p represses the proliferation, migration and invasion of OC cells by directly targeting TIAM1 and inactivating the Notch signaling pathway. These observations demonstrate that miR1271-5p/TIAM1 pair might be developed into a novel therapeutic regimen to better the outcomes and treatment for OC patients. 


\section{Abbreviations}

OC: Ovarian cancer; GEO: Gene Expression Omnibus; TCGA: The Cancer Genome Atlas; CCK-8: Cell counting kit 8; GTEx: Genotype-Tissue Expression

\section{Acknowledgements}

Not applicable.

\section{Authors' contributions}

All authors contributed to data analysis, drafting or revising the article, gave final approval of the version to be published, and agree to be accountable for all aspects of the work.

\section{Funding}

This study was supported by Heilongjiang Provincial Science Fund Project (Grant.no. ZD2018020) and Heilongjiang University of Traditional Chinese Medicine Graduate Innovation Research Project (Grant.no.2019yjscx007).

\section{Availability of data and materials}

The analyzed data sets generated during the study are available from the corresponding author on reasonable request.

\section{Ethics approval and consent to participate}

Not applicable.

\section{Consent for publication}

Not applicable.

\section{Competing interests}

The authors declare that they have no competing interests.

\section{Author details}

'Department of Gynecology, The First Affiliated Hospital of Heilongjiang University of Traditional Chinese Medicine, Harbin 150040, Heilongjiang, China. ${ }^{2}$ Heilongjiang University of Traditional Chinese Medicine, First Clinical Medical College, Harbin 150040, Heilongjiang, China. ${ }^{3}$ Department of Chinese medicine, Harbin Institute of Technology Hospital, Harbin 150006, Heilongjiang, China. ${ }^{4}$ Heilongjiang University of Traditional Chinese Medicine, College of Pharmacy, No. 24, Heping Road, Xiangfang District, Harbin 150040, Heilongjiang Province, China.

\section{Received: 23 April 2020 Accepted: 14 September 2020}

Published online: 18 September 2020

\section{References}

1. Liu B, et al. Ovarian cancer immunotherapy: opportunities, progresses and challenges. J Hematol Oncol. 2010;3:7.

2. Ottevanger PB. Ovarian cancer stem cells more questions than answers. Semin Cancer Biol. 2017:44:67-71.

3. Eisenhauer EA. Real-world evidence in the treatment of ovarian cancer. Ann Oncol. 2017;28(suppl_8):viii61-5.

4. Torre LA, Trabert B, DeSantis CE. Ovarian cancer statistics, 2018. CA Cancer J Clin. 2018;68(4):284-96.

5. Sun Z, et al. Effect of exosomal miRNA on cancer biology and clinical applications. Mol Cancer. 2018;17(1):147.

6. Di Leva G, Garofalo M, Croce CM. MicroRNAs in cancer. Annu Rev Pathol. 2014:9:287-314

7. Zhang L, et al. MiR-212-3p suppresses high-grade serous ovarian cancer progression by directly targeting MAP 3K3. Am J Transl Res. 2020;12(3):875-88.

8. Choi PW, et al. MicroRNA-200 family governs ovarian inclusion cyst formation and mode of ovarian cancer spread; 2020.

9. Paliwal N, Vashist M. Evaluation of miR-22 and miR-21 as diagnostic biomarkers in patients with epithelial ovarian cancer. 3 Biotech. 2020;10(3):142.

10. Li $Y$, et al. Mir-30b-3p affects the migration and invasion function of ovarian cancer cells by targeting the CTHRC1 gene. Biol Res. 2020;53(1):10.

11. Liu N, et al. Long non-coding RNA MALAT1 facilitates the tumorigenesis, invasion and glycolysis of multiple myeloma via miR-1271-5p/SOX13 axis. J Cancer Res Clin Oncol. 2020;146(2):367-79.

12. Wang $Y, X u L$, Jiang $L$. miR-1271 promotes non-small-cell lung cancer cell proliferation and invasion via targeting HOXA5. Biochem Biophys Res Commun. 2015;458(3):714-9.
13. Wang $L$, et al. A ten-microRNA signature identified from a genome-wide microRNA expression profiling in human epithelial ovarian cancer. PLoS One. 2014;9(5):e96472.

14. Chiu CY, et al. Cloning and characterization of T-cell lymphoma invasion and metastasis 2 (TIAM2), a novel guanine nucleotide exchange factor related to TIAM1. Genomics. 1999;61(1):66-73.

15. Li Z, et al. By downregulating TIAM1 expression, microRNA-329 suppresses gastric cancer invasion and growth. Oncotarget. 2015;6(19):17559-69.

16. Engers $R$, et al. Prognostic relevance of Tiam1 protein expression in prostate carcinomas. Br J Cancer. 2006;95(8):1081-6.

17. Xu K, et al. The fibroblast Tiam1-osteopontin pathway modulates breast cancer invasion and metastasis. Breast Cancer Res. 2016;18(1):14.

18. Li H, et al. Clinical implication of Tiam1 overexpression in the prognosis of patients with serous ovarian carcinoma. Oncol Lett. 2016;12(5):3492-8.

19. Li J, et al. Tiam1, negatively regulated by miR-22, miR-183 and miR-31, is involved in migration, invasion and viability of ovarian cancer cells. Oncol Rep. 2012;27(6):1835-42.

20. Wang $Z$, et al. Emerging role of notch in stem cells and cancer. Cancer Lett. 2009;279(1):8-12.

21. Colombo M, et al. Notch-directed microenvironment reprogramming in myeloma: a single path to multiple outcomes. Leukemia. 2013;27(5):1009-18.

22. Shawber CJ, et al. Notch signaling in primary endothelial cells. Ann N Y Acad Sci. 2003:995:162-70.

23. Cheng P, et al. Effects of notch signaling on regulation of myeloid cell differentiation in cancer. Cancer Res. 2014;74(1):141-52.

24. Wang $Z$, et al. Cross-talk between miRNA and notch signaling pathways in tumor development and progression. Cancer Lett. 2010;292(2):141-8.

25. Liu HM, et al. MicroRNA-1271-5p inhibits cell proliferation and enhances radiosensitivity by targeting CDK1 in hepatocellular carcinoma. J Biochem. 2019:167:513-24.

26. Yang Y, Chen L. Downregulation of IncRNA UCA1 facilitates apoptosis and reduces proliferation in multiple myeloma via regulation of the miR-12715p/HGF axis. J Chin Med Assoc. 2019:82(9):699-709.

27. Chen $Y$, et al. MicroRNA-1271 inhibits the progression of papillary thyroid carcinoma by targeting IRS1 and inactivating AKT pathway. Eur Rev Med Pharmacol Sci. 2019;23(18):7989-99.

28. Jiao $Y$, et al. miR-1271 inhibits growth, invasion and epithelial-mesenchymal transition by targeting ZEB1 in ovarian cancer cells. Onco Targets Ther. 2019:12:6973-80.

29. Rose SL, et al. Notch 1 signaling is active in ovarian cancer. Gynecol Oncol. 2010;117(1):130-3.

30. Tian F, et al. Salidroside protects LPS-induced injury in human thyroid follicular epithelial cells by upregulation of MiR-27a. Life Sci. 2018;213:1-8.

31. Sun $Q$, et al. MicroRNA-139-5P inhibits human prostate cancer cell proliferation by targeting Notch1. Oncol Lett. 2018;16(1):793-800.

32. Liang $\mathrm{T}$, et al. MicroRNA-433 inhibits migration and invasion of ovarian cancer cells via targeting Notch1. Neoplasma. 2016;63(5):696-704.

33. Liu MX, et al. Epigenetic silencing of microRNA-199b-5p is associated with acquired chemoresistance via activation of JAG1-Notch1 signaling in ovarian cancer. Oncotarget. 2014;5(4):944-58.

34. Feng $Z$, et al. Inhibition of gamma-secretase in Notch1 signaling pathway as a novel treatment for ovarian cancer. Oncotarget. 2017;8(5):8215-25.

35. Alniaimi AN, et al. Increased Notch1 expression is associated with poor overall survival in patients with ovarian cancer. Int J Gynecol Cancer. 2015; 25(2):208-13.

36. Dong C, et al. Long non-coding RNA HOTAIR regulates proliferation and invasion via activating notch signalling pathway in retinoblastoma. J Biosci. 2016;41(4):677-87.

37. Flores AN, et al. NUMB inhibition of NOTCH signalling as a therapeutic target in prostate cancer. Nat Rev Urol. 2014;11(9):499-507.

\section{Publisher's Note}

Springer Nature remains neutral with regard to jurisdictional claims in published maps and institutional affiliations. 